\title{
Serotypes and Antimicrobial Susceptibility Patterns of Nasopharyngeal Pneumococci Isolated from HIV-Infected Children in Selected Pediatric Clinics in Nairobi, Kenya
}

\author{
Catherine N. Mwangi' ${ }^{1}$, Gunturu Revathi' ${ }^{2}$, Anne W. Muigai' ${ }^{1}$, Samuel Kariuki ${ }^{3}$ \\ ${ }^{1}$ Jomo Kenyatta University of Agriculture and Technology, Nairobi, Kenya \\ ${ }^{2}$ Aga Khan University Hospital, Nairobi, Kenya \\ ${ }^{3}$ Kenya Medical Research Institute, Nairobi, Kenya \\ Email:cmwangi99@gmail.com, guntururevathi@aku.edu, annemuigai@yahoo.com, \\ samkariuki2@gmail.com
}

Received 1 February 2016; accepted 28 March 2016; published 31 March 2016

Copyright (C) 2016 by authors and Scientific Research Publishing Inc.

This work is licensed under the Creative Commons Attribution International License (CC BY). http://creativecommons.org/licenses/by/4.0/

(c) () Open Access

\section{Abstract}

Background: Streptococcus pneumoniae (pneumococcus) is one of the most frequent causes of bacterial infection in children and is a leading cause of otitis, sinusitis, pneumonia, and meningitis worldwide. Nasopharyngeal colonization is a risk factor for pneumococcal disease, a leading cause of complications and death in infants. HIV-infected persons are at high risk of invasive pneumococcal disease. Method: Nasopharyngeal swabs were collected from 296 HIV infected children below five years recruited from Gertrude's Children hospital and Nazareth Hospital Nairobi, Kenya. The nasopharyngeal swabs were processed to isolate $S$. pneumoniae, which were serotyped and tested for drug susceptibility. Results: The carriage prevalence of $S$. pneumoniae in the study was $30.4 \%$ while the isolated serotypes were (in order of decreasing frequency): 35B, 19F, 3, 13, 15A, 11A, 16F, 7C and 23A. Most of the serotypes were resistant to the commonly used antibiotics but all were susceptible to vancomycin and chloramphenicol. Conclusion: Carriage prevalence of nasopharyngeal $S$. pneumonia in HIV infected children was lower than that of similar prevalence studies in children. Most of the $S$. pneumoniae isolates were however non pneumococcal vaccine isolates.

\section{Keywords}

Streptococcus pneumoniae, Nasopharynx, Serotypes, HIV, Children, PCV 


\section{Introduction}

The aim of this study was to provide baseline information on the prevalence of nasopharyngeal pneumococcal carriage, serotypes and antimicrobial susceptibility patterns of these pneumococci in HIV infected children in Nairobi prior to the inclusion of pneumococcal conjugate vaccine to the routine childhood immunization programme in Kenya. Interest into pneumococcal disease has been rekindled by emergence of antibiotic resistance and recognition of the pneumococcus as a Human Immunodeficiency Virus (HIV)-associated pathogen [1]. HIV/AIDS being endemic in Kenya and pneumococci being the leading bacterial infection in HIV positive individuals especially children, pneumococci infections should be of great concern and studies should be undertaken

This study exploited the asymptomatic nasopharyngeal carriage of $S$. pneumoniae which is widely prevalent in young children and has been related to the development of disease and the spread of the pathogen. Hence the collection of nasopharyngeal swabs helps to monitor the circulating serotypes and emergence of new drug resistant strains in Nairobi community prior introduction of the new pneumococcal vaccine (Synflorix ${ }^{\mathrm{TM}}$ ). This in turn enables us to predict the antimicrobial resistance patterns and the likely effectiveness of the pneumococcal vaccine.

\section{Method}

\subsection{Study Design and Population}

The study is a descriptive, Clinico-laboratory based, cross sectional study, carried for a period of four months between November 2009 and March 2010. The study population consisted HIV infected children below the age of five years, recruited from Gaterude's children Hospital and Nazareth Hospital located within Nairobi, presented for routine well care whose parents/guardians consent had been given.

\subsection{Ethical Consideration}

This study project was approved for implementation by the KEMRI Scientific Steering Committee (SSC No. 1629) and the National Ethics Review Committee.

\subsection{Inclusion Criteria}

- Non-vaccinated HIV seropositive children below five years whose parental/guardian consent had been given.

\subsection{Exclusion Criteria}

- HIV seronegative children

- HIV seropositive children above five years of age

- HIV seropositive children whose parental consent had not been given.

\subsection{Sample Size and Sampling Procedure}

A sample size of 296 HIV seropositive children was reached using statistical formula, Cochran (1963) developed to yield a representative sample for proportions,

$$
n_{0}=\frac{Z^{2} P q}{e^{2}}
$$

where $n_{0}$ is the sample size.

$Z^{2}$ is the abscissa of the normal curve that cuts off an area at the tails (1-equals the desired confidence level, e.g., $95 \%)^{1}, Z=1.96$.

$e$ is the desired level of precision or sometimes called sampling error is $\pm 5 \%$.

$P(26 \%)$ is the estimated prevalence of nasopharyngeal pneumococcal carriage in HIV positive children in a previous study in Nairobi, Kenya by Rusen et al., 1997.

$q$ is $1-p$.

\subsection{Subject Recruitment}

The parent/guardian of the children was counseled on the type of the study to be done and the benefits of the 
study. Questionnaires were filled from interviewing the parents who gave consent to the study.

\subsection{Sample Collection}

Swabs were collected by trained medical personnel (physicians and nurses) where WHO working group guidelines on nasopharyngeal studies of $S$. pneumoniae, were followed [2]. The subject's head was tipped slightly backward and the swab passed directly backwards, parallel to the floor of the nasopharynx.

The swab was passed through the nostril till it reached the posterior pharynx which is approximately two-thirds the distance from the nostril to the ear lobe. Where resistance was encountered, the swab was removed and attempt made to pass the swab through the other nostril. Once in place, it was rotated through 180 degrees and left in place for about 2 seconds to saturate the tip before removing it slowly.

Once the nasopharyngeal (NP) specimen was collected the wire tips were cut off using wire cutters and put in the vials containing $1.0 \mathrm{ml}$ of STGG transport medium and labeled with the study number.

The specimen was maintained and transported on wet ice to the KEMRI lab within eight hours. The swab used was of pediatric size with a rayon tip and a flexible aluminium shaft from Medical Wire and Equipment Company, Town, UK.

After swabbing, the parents/guardians were asked to answer the questions according to the questionnaire.

\subsection{Processing of the Nasopharyngeal Swabs}

Upon arrival of the swab samples in the laboratory, the fresh swabs were vortexed at 2500 revolutions for 20 seconds using vortex machine. Using a micropipette $10 \mu \mathrm{l}$ of sample was aspirated onto prior labeled blood agar media plate of Columbia agar with $5 \%$ sheep blood. Sample was then streaked using a sterilized nichrome wire loop onto half blood agar primary plate. The plates were incubated at $37^{\circ} \mathrm{C}$ in $5 \% \mathrm{CO}_{2}$ overnight.

From the primary plate four presumptive pneumococcal colonies that were morphologically consistent with $S$. pneumoniae, showing small, grey and mucoid colonies surrounded by a greenish zone of haemolysis were picked.

They were streaked out on four halves of blood agar plates, so as to select colonies that had different morphology distinguished from viridians and other Streptococcus species by Optochin and bile solubility testing.

\subsection{Optochin Susceptibility Testing}

A 6-mm, $5 \mu$ g optochin disk (Becton Dickinson) was aseptically paced on the centre of the streak where the inoculum was densest and incubated at $37^{\circ} \mathrm{C}$ in $5 \% \mathrm{CO}_{2}$ overnight. Optochin susceptible strains were identified as S. pneumoniae with zones of inhibition greater than $14 \mathrm{~mm}$. 7 to $13 \mathrm{~mm}$ were intermediate and less than $7 \mathrm{~mm}$ were resistant.

Isolates that were optochin susceptible were considered pneumococci; those of intermediate susceptibility were tested for bile solubility. Those that were optochin-resistant were considered to be species other than pneumococci.

\subsection{Bile Susceptibility Testing by Tube Method}

Bile susceptibility testing was performed for further characterization and identification of $\alpha$-hemolytic strains with zones of inhibition ranging between $7 \mathrm{~mm}$ and $13 \mathrm{~mm}$. A loop of the suspected strain from optochin test plate was suspended in $0.5 \mathrm{ml}$ saline similar to 0.5 McFarland turbidity standards. The suspension was then divided into two equal amounts $0.25 \mathrm{ml}$ each tube. $0.25 \mathrm{ml}$ of saline was added in one tube and $0.25 \mathrm{ml}$ of $2 \%$ sodium desoxycholate/bile salts (Oxoid). Tube containing bile was examined periodically for lysis; strains that yielded clearing of suspension were reported bile soluble i.e. S. pneumoniae.

\subsection{Serotyping}

Serotyping was carried out in Kenya Medical Research Institute in collaboration with Welcome Trust Research Laboratories (KEMRI/WTRL) Kilifi district. Pneumococci were serogrouped by latex agglutination as described below and the serotypes defined by Quellung Reaction [3].

\subsection{Latex Agglutination Test}

A cell suspension was prepared in three to four drops (200 - $300 \mu \mathrm{l})$ of PBS pH 7.2 with half loop, full of the 
bacterial growth. On a glass slide $5 \mu$ of latex reagents (Dryspot ${ }^{\mathrm{TM}}$ Pneumo Oxoid) and $10 \mu \mathrm{l}$ of cell suspension (ratio 1:2) was mixed and the slide rocked for one minute.

A visible clumping of the suspension was considered as a positive result while a negative result remained smooth.

Ten microlitres of the cell suspension were placed at two points on the slide and two pools were tested at a time by adding $5 \mu \mathrm{l}$ of the respective latex reagents to each and stirring with a pipette tip to mix well. This was done till the specific pool was known. Once the pool was known, each type or group within the pool was tested individually.

The types that historically occurred more often were tested first and followed by the next ones in the order of their occurrence. For example in pool B, type 19 was checked first then followed by 6 etc. and in pool H, 23 and 15. All the pneumococcal strains were then identified finally by the Quellung test.

\subsection{Quellung Test}

The cell suspension prepared above was mixed at equal volumes with the respective factor sera (BBL ${ }^{\mathrm{TM}}$ Pneumoslide ${ }^{\mathrm{TM}}$ ) to determine its factors. $5 \mu \mathrm{l}$ of the cell suspension and $5 \mu \mathrm{l}$ of the antiserum factor reagents (ratio 1:1) were mixed with a pipette tip. This was covered with a No. 1 cover slip and examined under an oil immersion objective lens. This was to check for the presence of apparent capsular swelling to identify the pneumococcal factors.

\subsection{Disk Diffusion Test}

Identified S. pneumococci isolates susceptibility was tested to a panel of 10 antibiotics by using disk diffusion techniques following guidelines of CLSI 2008. A loop of the suspected strain from purity plate was suspended in $0.5 \mathrm{ml}$ saline similar to $0.5 \mathrm{McF}$ arland turbidity standards. A swab was dipped into the suspension and excess suspension fluid drained by pressing on the side of the tube. Mueller agar $\left(\mathrm{Oxoid}^{\mathrm{TM}}\right)$ was mixed with sheep blood plates were swabbed with this cell suspension. Antibiotic embedded disks were then carefully placed on the placed and incubated for 18 hours in a $\mathrm{CO}_{2}$ incubator. Susceptible bacteria to a particular antibiotic showed an area of clearing around the antibiotic disk called zone of inhibition. Larger zones correlate with smaller minimum inhibitory concentration of an antibiotic.

Inoculums of fresh pure cultures of test $S$. pneumoniae (grown overnight on blood agar) and QC strain $S$. pneumoniae ATCC49619 were suspended in sterile physiological saline equal to 0.5 Mc Fahrland turbidity standard. The suspension was used within 15 minutes to avoid over growth of the bacteria. Sterilized Mueller Hinton agar $\left(\mathrm{Oxoid}^{\mathrm{TM}}\right)$ supplemented with 5\% sheep agar was poured onto the agar plates and allowed to cool before swabbing the surface with the samples.

A swab dipped into the suspension was used to inoculate entire surface of Mueller Hinton agar (Oxoid ${ }^{\mathrm{TM}}$ ) with $5 \%$ sheep agar plate three times while rotating the plate at 60 degrees between each inoculation. The plate was left to dry for few minutes before placing the antibiotic disks using forceps and incubated in a $5 \%$ carbon dioxide incubator for $20-24$ hours at $35^{\circ} \mathrm{C}$. After overnight incubation, the zones of inhibition were measured using a ruler and results recorded in millimeters $(\mathrm{mm})$. These results were compared to the CLSI standard zone sizes.

\subsection{E-Test for Minimum Inhibitory Concentration}

E-test for S. pneumoniae was used to distinguish complete and intermediate resistance. It was also used to quantify the results of the Oxacillin disk diffusion test by performing MIC testing of the antibiotics. Viable colonies from overnight blood agar plates were suspended into a broth tube to achieve a bacterial suspension equivalent to a 0.5 McFarland turbidity standard. A cotton swab was dipped into the bacterial suspension, pressed onto the side of tube to drain excess fluid. Entire surface of Mueller Hinton agar (Oxoid ${ }^{\mathrm{TM}}$ ) with $5 \%$ sheep blood was swabbed three times each at 60 degree angle and plate is allowed to dry for few minutes.

E-test ${ }^{\circledR}$ (AB biodisk) strips were removed then carefully placed on the dried inoculated agar plates with the printed MIC values facing upward using an E-test applicator. Plates were then incubated in an inverted position in a $5 \%$ carbon dioxide incubator for $20-24$ hours at $35^{\circ} \mathrm{C}$. Quality control was performed on a daily basis using S. pneumoniae ATCC 49619. MICs were read from the intersection of eclipse formed zone of inhibition with the 
value printed on the E-test strip and recorded. Since E-test generates MICs from a continuous gradient, values in between the two-fold dilutions can be obtained. In such a case the values were rounded up to the two-fold dilutions before susceptibility categorization.

\subsection{Data Analysis}

Descriptive epidemiology was used to describe carriage prevalence of S. pneumoniae and their antibiotic susceptibility patterns. Raw data was analyzed using SPSS version 10. The frequency of the variables was calculated to exclude any missing values to minimize the effects of the outcome. Unilateral analysis of the variables was used to identify potential risk factors after which logistic regression was carried out. Logistic regression was used to find association between each risk factor and nasopharyngeal carriage prevalence of S. pneumoniae. Logistic regression was chosen due to the fact that the dependent variable (carriage prevalence) has a binary outcome (carrier or non carrier). Logistic regression is an effective way to determine the relative importance of the independent variables. $\mathrm{P}$ values and $95 \%$ confidence Intervals were calculated to determine statistical significance.

\section{Results}

A total of 296 samples were collected from recruited children and the average age of the children recruited was 30.2 months. The study population was composed of $55.1 \%$ males and $44.9 \%$ females (Table 1 ). Age group 13 to 24 months had the highest number of males at 38 while less than twelve months and 25 to 36 months age group had the highest number of females at 31 (Table 1).

A total of 94 isolates were recovered from the nasopharyngeal swab samples collected during the study. These 94 isolates were from 90 children recruited in the study where the age group of less than twelve months and 49-60 months had the highest colonization of $23 \%$ as shown in Table 2.

The carriage prevalence of $S$. pneumoniae among the children was $30.4 \%$ (Table 2). The prevalence of the males and that of females was $30.6 \%$ and $30.1 \%$ respectively. The average carriage rate for the different age groups was $31.14 \%$.

No significant difference was found in carriage prevalence between male and female children in this study; their numbers had the strength to test any variation in carriage between the sexes (Figure 1). Males in the 37 to 48 months age group had the highest prevalence at $81.3 \%$ (Table 2). The females had the highest prevalence in the 13 to 24 months age group; this was the lowest prevalence for the males (Table 2). With p value 0.33653 , df 89 and $\alpha=0.05$, there was no significant difference between the males and females.

Ten different serotypes of the nasopharyngeal isolates were collected, with decreasing frequency in prevalence; 35B, 3, 19F, 10A, 13, 15A, 11A, 7C, 16F and 23A. Serotype 35B isolates had the highest frequency of $24.4 \%$ among the nasopharyngeal pneumococci in this study while serotype 3 and $19 \mathrm{~F}$ were the second and third most prevalent isolates at $17 \%$ and $15.8 \%$ as shown in Figure 2. Of the 94 serotypes, 16 (17\%) were serotype 3, 15 (15.8\%) were serotype 19F, 14 (14.8\%) were serotype 13, 7 (7.4\%) were serotype 11A and 15A, 5 (5.3\%)

Table 1. Age and sex of children recruited for the study.

\begin{tabular}{ccccccccc}
\multirow{2}{*}{ SEX } & \multicolumn{9}{c}{ AGE GROUP } & TOTAL & PERCENTAGE \\
\cline { 2 - 6 } & $<12$ & $13-24$ & $25-36$ & $37-48$ & $49-60$ & 36 & 163 & 55.06 \\
MALE & 34 & 38 & 34 & 21 & 19 & 26 & 133 & 44.94 \\
FEMALE & 31 & 26 & 31 & 40 & 62 & 296 & 100 \\
TOTAL & 65 & 64 & 65 & 400 \\
\hline
\end{tabular}

Table 2. Age group and sex specific prevalence of Streptococcus pneumoniae carriage.

\begin{tabular}{|c|c|c|c|c|c|c|c|c|c|c|}
\hline \multirow[b]{3}{*}{ MALES } & \multicolumn{10}{|c|}{ AGE GROUP CARRIAGE } \\
\hline & \multicolumn{2}{|c|}{$<12$} & \multicolumn{2}{|c|}{$13-24$} & \multicolumn{2}{|c|}{$25-36$} & \multicolumn{2}{|c|}{$37-48$} & \multicolumn{2}{|c|}{$49-60$} \\
\hline & 10 & $47.6 \%$ & 8 & $42.1 \%$ & 8 & $61.5 \%$ & 13 & $81.3 \%$ & 11 & $52.3 \%$ \\
\hline FEMALES & 11 & $52.3 \%$ & 11 & $57.9 \%$ & 5 & $38.5 \%$ & 3 & $18.7 \%$ & 10 & $47.6 \%$ \\
\hline
\end{tabular}




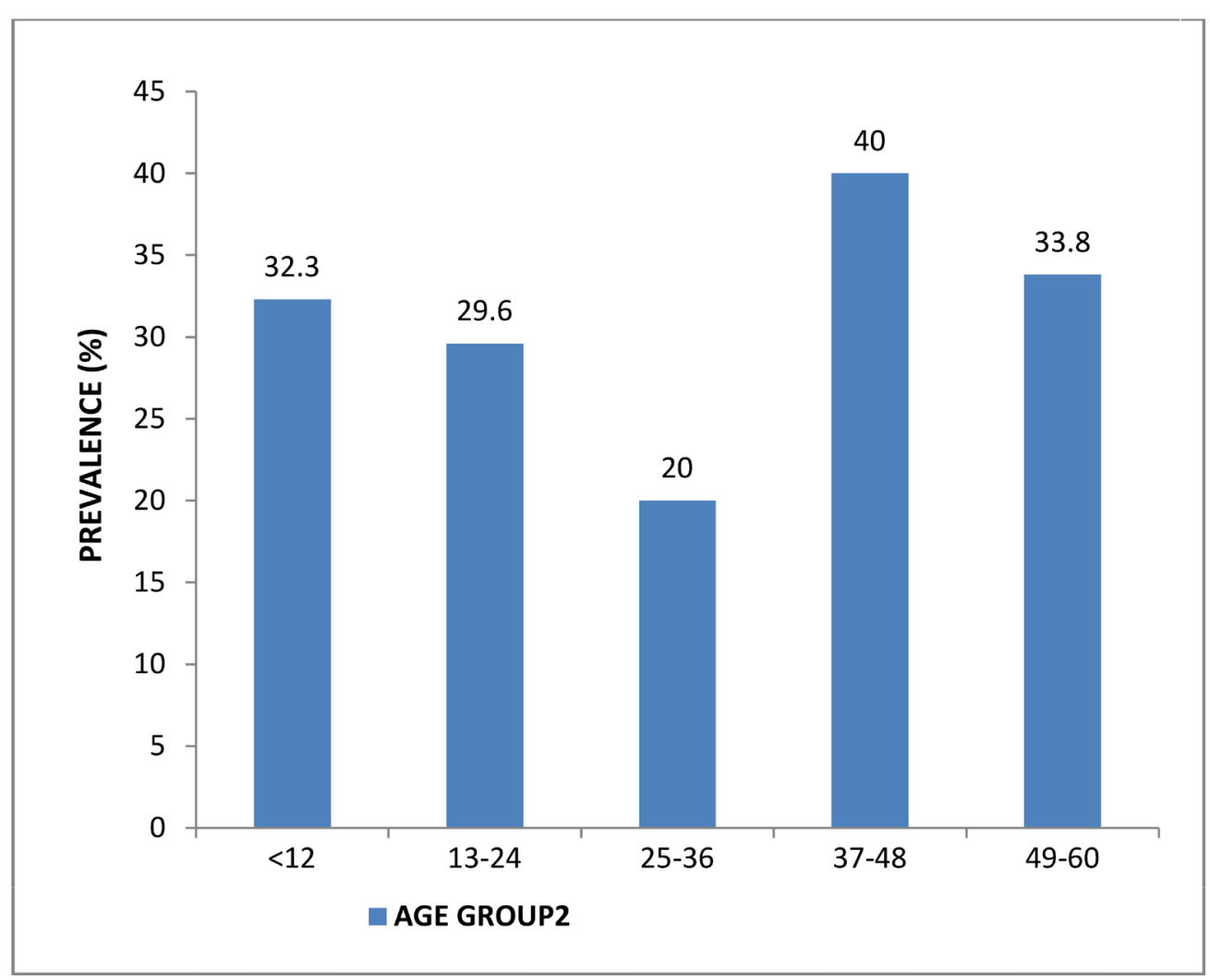

Figure 1. Streptococcus pneumoniae carriage rate by age group.

were serotype $10 \mathrm{~A}$ and 3 (3.2) were serotype 7C and 16F.

Resistance rate to common antibiotics used in treatment such as cefotaxime and Amoxicillin was $2.4 \%$, and $6.6 \%$ respectively. Resistance of Streptococcus pneumoniae to cotrimoxazole were $55.5 \%$, while $26.8 \%$ of the isolates were intermediately resistant to cotrimoxazole (Figure 3). Resistance rate to erythromycin was $17.7 \%$ while 13.3\% of the Streptococcus pneumoniae isolates showed intermediate resistance. All the Streptococcus pneumoniae were resistant to oxacillin while $49 \%$ of the isolates were resistant penicillin. Intermediate resistance to penicillin was shown by $11 \%$ of Streptococcus pneumoniae (Figure 3). All the S. pneumoniae isolates were susceptible to vancomycin and chloramphenicol while $91.1 \%$ were resistant to sulphtrimethoprim. $S$. pneumoniae resistance rates to amoxicillin (6.6\%), ceftriaxone $(6.7 \%)$ and levofloxacin $(5.5 \%)$ were low (Figure 3). Streptococcus pneumoniae had resistance to Clindamycin (24.4\%), Cefotaxime (2.4\%) and Ofloxacin (13.3\%)

Serotype 35B is a non invasive, non vaccine serotype which contributed to $43.5 \%$ of penicillin resistance among the isolates (Table 3). Other non invasive non vaccine serotypes $15 \mathrm{~A}$ and $11 \mathrm{~A}$ had penicillin resistance of $7.1 \%$. The predominant serotypes among co-trimoxazole resistant isolates were 35B, 19F and 11A with frequencies of $80.5 \%, 53.3 \%$ and $85.7 \%$ (Table 4 ).

The 37 to 48 months age group serotype 35B had the highest frequency of $43.8 \%$ and least frequent of $9.5 \%$ in the 49 to 60 months age group. Serotype 3 was the second predominant serotype and had the highest frequency of $28.6 \%$ at age group 49 to 60 months as shown in Table 5. Serotype 19F was the most prevalent isolate at $15.8 \%$ while $23 \mathrm{~A}$ was the least prevalent at $1 \%$ (Table 5 ).

\section{Discussion}

The carriage prevalence for S. pneumoniae among the children in this study was $30.4 \%$, while the carriage prevalence in a study conducted in Kilifi was 53\% [4]. This was also lower than a study in Netherlands where the carriage prevalence was $47 \%$ [5].

Carriage prevalence was highest between the ages of 37 and 60 months at $44.5 \%$, this may have been attri- 
buted to the very frequent and close contact between them and other children in school/day care centers their younger siblings who are often carriers. We found that the presence of siblings under 5 years was a risk factor ( $\mathrm{p}$ $=0.001$; odds ratio, 3.027 ; $95 \%$ confidence interval 1.535 to 5.97 ) contributing to nasopharyngeal $S$. pneumoniae carriage. This was similar to a study in Turkey where, having a sibling under five years of age was found to be a significant risk factor $(\mathrm{p}=0.03$, odds ratio, 4.6) for carriage of $S$. pneumoniae [6]. It is a risk factor as transmission occur secondary to direct person to person contact, by means of respiratory droplets or through oral contact and items freshly soiled with respiratory droplets. Children are more prone to colonization of pneumococci compared to adults whose decline of carriage could reflect acquisition of local mucosal immunity as a result of repeated colonization by many different [7].

Contrary to other studies, serotype $35 \mathrm{~B}$ was the most predominant at $24.5 \%$ while serotype $23 \mathrm{~A}$ was the least predominant at $1.1 \%$. In a study in Netherland, the serotype $19 \mathrm{~F}$ was the most predominant with a prevalence of $19 \%$ [5] while in a 2008 study in New Zealand, the prevalence of serotype 19F was higher at $23.6 \%$. Serotype 3 had low prevalence compared to this study at $4 \%$. Serotype $19 \mathrm{~F}$ which is a PCV7 and PHiD-CV10 (Synflorix ${ }^{\mathrm{TM}}$ ) vaccine serotype, ranked third in the nasopharyngeal isolates and constituted $14.8 \%$ of all the isolates. This is lower compared to PCV-7 (26\%) and PHiD-CV10 (26\%) vaccine serotypes isolated from Aboriginal children in Australia [8]. But when compared with PCV13 and PPV23 vaccine serotypes in Australia which was 26\%, the nasopharyngeal serotypes in this study had higher frequency of vaccine serotypes at $31.8 \%$ and $44.4 \%$ respectively (Table 6). These vaccine serotypes included 3, 10A, 11A and 19F hence the wide margin for the PCV13 and PPV 23 vaccine as compared to PHiD-CV10 (Synflorix ${ }^{\mathrm{TM}}$ ) vaccine serotypes. The results indicate that PCV7 and PCV10 would provide less coverage against the nasopharyngeal pneumococci as compared to PCV13 and PCV23. However PCV23 vaccine fails to induce immune memory in children $\leq 2$ years hence not appropriate for routine childhood immunization [9].

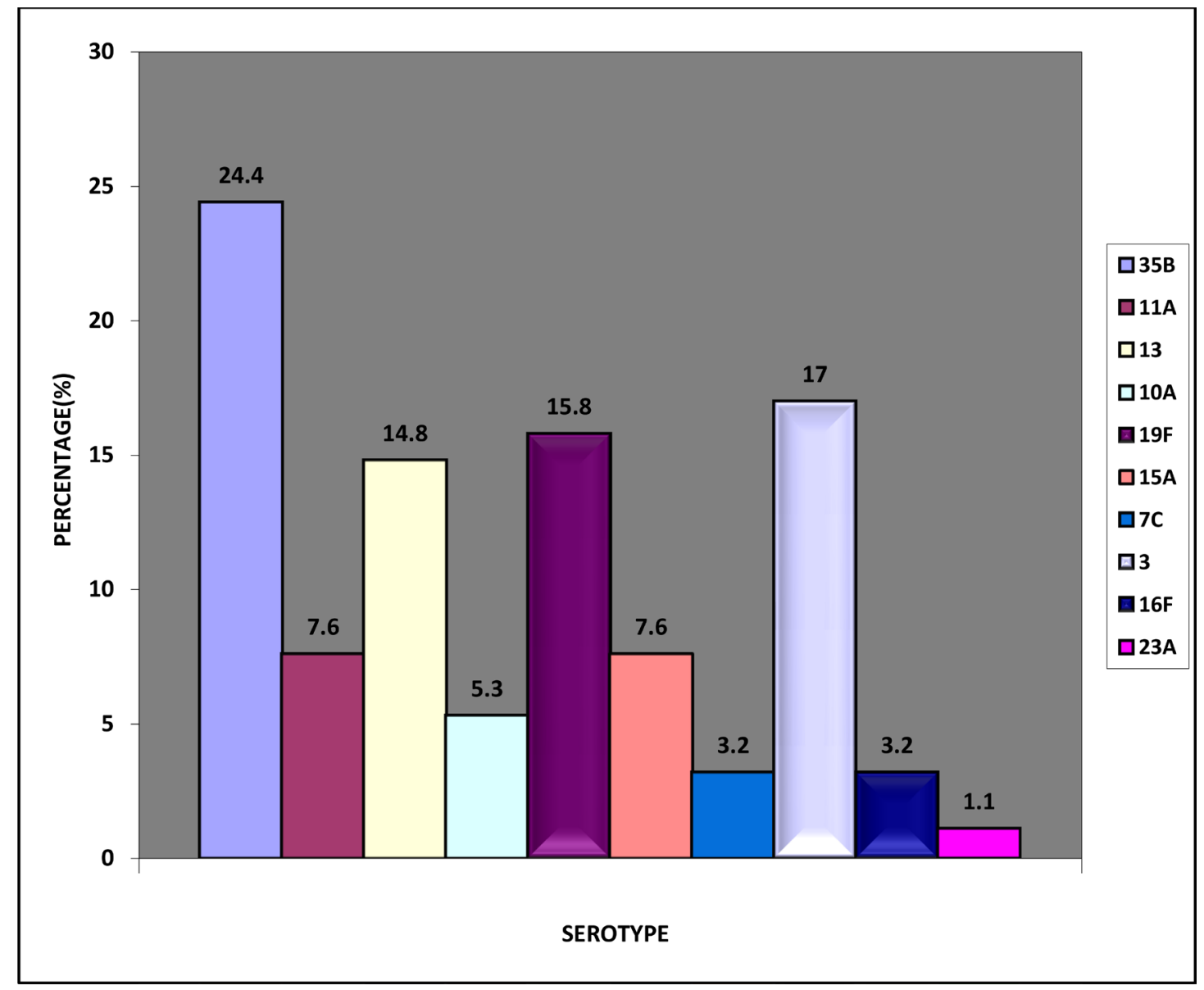

Figure 2. Streptococcus pneumoniae serotype distribution by percentage. 


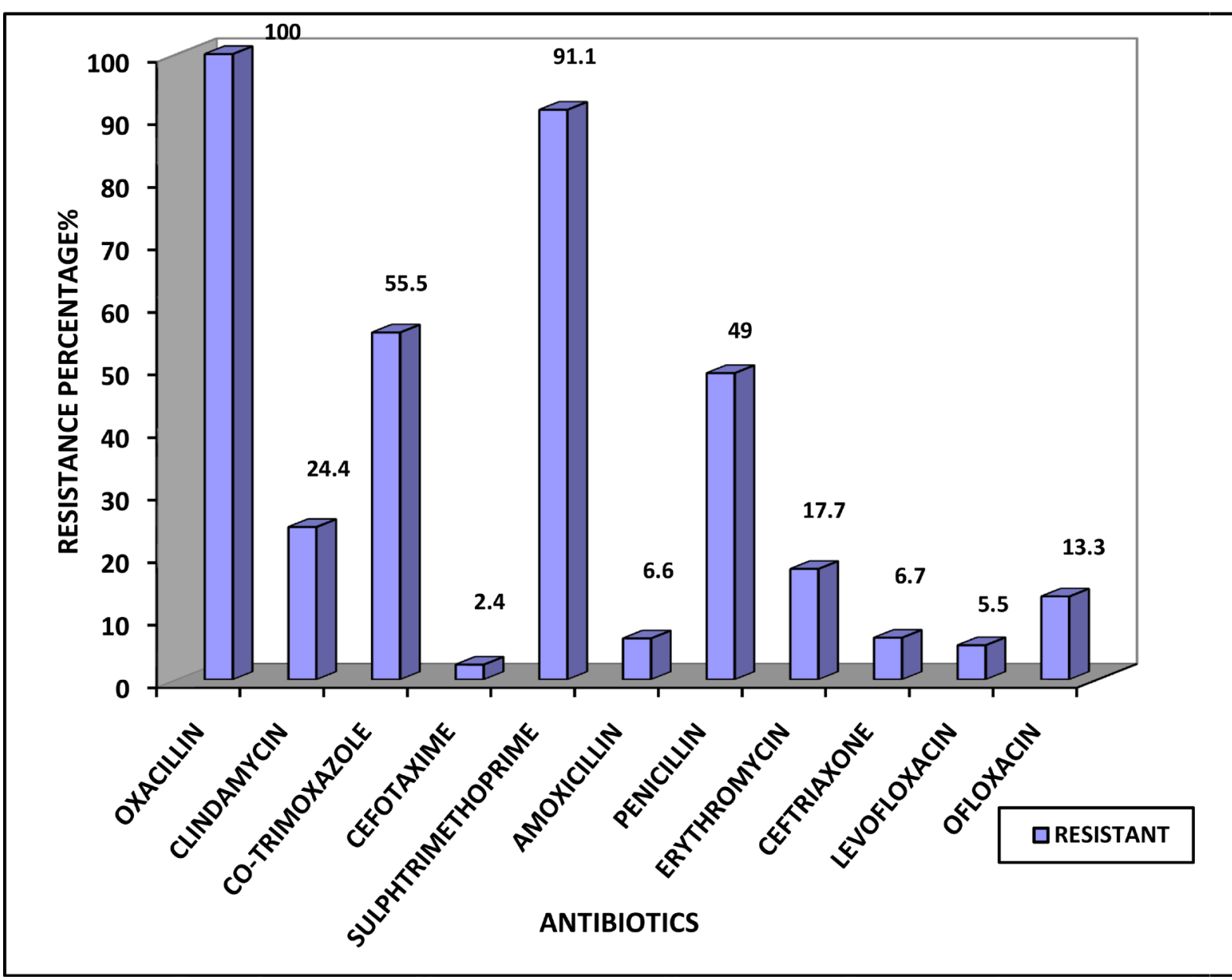

Figure 3. Resistant patterns of the nasopharyngeal Streptococcus pneumonia.

Table 3. Invasive serotypes distribution.

\begin{tabular}{ccc}
\hline INVASIVE SEROTYPE & TOTAL $\mathbf{n}=\mathbf{9 4}$ & PERCENTAGE $\%$ \\
\hline $19 \mathrm{~F}$ & 15 & 15.8 \\
$23 \mathrm{~A}$ & 1 & 1 \\
$7 \mathrm{C}$ & 3 & 3.2 \\
3 & 16 & 17 \\
TOTAL & 35 & 37.2 \\
\hline
\end{tabular}

The 49 to 60 months age group had the highest PCV13 and PCV23 vaccine serotype coverage when compared to the other age groups. This may be due to exposure to other children in day care centre where transmission of $S$. pneumoniae among children in is high. The results indicate that childhood pneumococcal vaccination would be expected to have a marked impact on both non-invasive and invasive pneumococcal infections. This is due to most of the resistance among the pneumococci is associated with serotype 19F which is a PHiD-CV10 vaccine serotype.

PHiD-CV10 vaccine serotypes cover major serotypes that have been isolated in East Africa. These serotypes are 1, 4, 5, 6A, 6B 14, 18C, 19F and 23F [10]. This was however not reflected in this study as only $14.8 \%$ of the isolated serotypes were covered by the new pneumococcal vaccine; PHiD-CV10 (Synflorix ${ }^{\mathrm{TM}}$ ). This could be of concern as emergence and spread of penicillin resistant and multidrug resistant non vaccine serotypes can contribute to drug resistant pneumococcal disease burden. 
Table 4. Antimicrobial resistance patterns of Streptococcus pneumoniae serotypes.

\begin{tabular}{cccccccccccc}
\hline & \multicolumn{1}{c}{ SEROTYPE PERCENTAGE (\%) } \\
\cline { 2 - 8 } ANTIBIOTICS & $35 \mathrm{~B}$ & $11 \mathrm{~A}$ & 13 & $10 \mathrm{~A}$ & $19 \mathrm{~F}$ & $15 \mathrm{~A}$ & $7 \mathrm{C}$ & 3 & $16 \mathrm{~F}$ & $23 \mathrm{~A}$ \\
& $\mathrm{n}=23$ & $\mathrm{n}=7$ & $\mathrm{n}=14$ & $\mathrm{n}=5$ & $\mathrm{n}=15$ & $\mathrm{n}=7$ & $\mathrm{n}=3$ & $\mathrm{n}=16$ & $\mathrm{n}=3$ & $\mathrm{n}=1$ \\
\hline Oxacillin & 100 & 100 & 100 & 100 & 100 & 100 & 100 & 100 & 100 & 100 \\
Vancomycin & 0 & 0 & 0 & 0 & 0 & 0 & 0 & 0 & 0 & 0 \\
Clindamycin & 26.1 & 28.6 & 28.6 & 40 & 26.7 & 28.6 & 0 & 18.8 & 0 & 0 \\
Ciprofloxacin & 0 & 0 & 0 & 0 & 0 & 0 & 0 & 0 & 0 & 0 \\
Cefotaxime & 3.2 & 1.4 & 0 & 0 & 20 & 0 & 0 & 0 & 0 & 0 \\
Sulphtrimethoprime & 82.6 & 100 & 85.7 & 100 & 93.3 & 100 & 100 & 92.3 & 100 & 100 \\
Cotrimoxazole & 60.8 & 85.7 & 50 & 80 & 60 & 28.6 & 66.7 & 38.5 & 66.7 & 0 \\
Amoxacillin & 0 & 0 & 14.3 & 20 & 0 & 0 & 33.3 & 0 & 0 & 0 \\
Benzylpenicillin & 43.5 & 57.1 & 57.1 & 60 & 73.3 & 57.1 & 66.7 & 81.3 & 100 & 0 \\
Erythromycin & 4.3 & 14.3 & 21.4 & 20 & 40 & 14.3 & 33.3 & 18.8 & 0 & 0 \\
Ceftriaxone & 8.7 & 0 & 7.1 & 0 & 20 & 0 & 0 & 0 & 0 & 0 \\
Levofloxacin & 13 & 0 & 7.1 & 0 & 0 & 0 & 0 & 44.5 & 0 & 0 \\
Ofloxacin & 21.7 & 0 & 7.1 & 20 & 20 & 14.3 & 0 & 18.8 & 0 & 0 \\
Chloramphenicol & 0 & 0 & 0 & 0 & 0 & 0 & 0 & 0 & 0 & 0 \\
\hline
\end{tabular}

Table 5. Streptococcus pneumoniae serotype distribution by age group.

\begin{tabular}{|c|c|c|c|c|c|c|c|c|c|c|}
\hline \multirow{3}{*}{$\begin{array}{c}\text { SEROTYPE } \\
35 B\end{array}$} & \multicolumn{10}{|c|}{ AGE GROUP } \\
\hline & \multicolumn{2}{|c|}{$\begin{array}{c}\leq 12 \\
n=21\end{array}$} & \multicolumn{2}{|c|}{$\begin{array}{l}13-24 \\
n=19\end{array}$} & \multicolumn{2}{|c|}{$\begin{array}{c}25-36 \\
n=13\end{array}$} & \multicolumn{2}{|c|}{$\begin{array}{l}37-48 \\
n=16\end{array}$} & \multicolumn{2}{|c|}{$\begin{array}{c}49-60 \\
n=21\end{array}$} \\
\hline & 6 & $28.6 \%$ & 4 & $21.1 \%$ & 4 & $30.8 \%$ & 7 & $43.8 \%$ & 2 & $9.5 \%$ \\
\hline $11 \mathrm{~A}$ & 3 & $14.3 \%$ & 0 & $0 \%$ & 1 & $7.7 \%$ & 0 & $0 \%$ & 3 & $14.3 \%$ \\
\hline 13 & 1 & $4.8 \%$ & 2 & $10.5 \%$ & 1 & $7.7 \%$ & 5 & $31.3 \%$ & 5 & $23.8 \%$ \\
\hline $10 \mathrm{~A}$ & 1 & $4.8 \%$ & 2 & $10.5 \%$ & 1 & $7.7 \%$ & 1 & $6.3 \%$ & 0 & $0 \%$ \\
\hline $19 \mathrm{~F}$ & 3 & $14.3 \%$ & 1 & $5.3 \%$ & 3 & $23.1 \%$ & 3 & $18.8 \%$ & 5 & $23.8 \%$ \\
\hline $15 \mathrm{~A}$ & 2 & $9.5 \%$ & 2 & $10.5 \%$ & 0 & $0 \%$ & 1 & $6.3 \%$ & 2 & $9.5 \%$ \\
\hline 7C & 2 & $9.5 \%$ & 1 & $5.3 \%$ & 0 & $0 \%$ & 0 & $0 \%$ & 0 & $0 \%$ \\
\hline 3 & 1 & $4.8 \%$ & 5 & $26.3 \%$ & 2 & $15.4 \%$ & 2 & $12.5 \%$ & 6 & $28.6 \%$ \\
\hline $16 \mathrm{~F}$ & 2 & $4.8 \%$ & $0 \%$ & $0 \%$ & 0 & $0 \%$ & 0 & $0 \%$ & 1 & $4.8 \%$ \\
\hline $23 \mathrm{~A}$ & 0 & $0 \%$ & $0 \%$ & $0 \%$ & 1 & $7.7 \%$ & 0 & $0 \%$ & 0 & $0 \%$ \\
\hline
\end{tabular}

Table 6. Streptococcus pneumoniae isolates covered by vaccine serotypes.

\begin{tabular}{cccc}
\hline VACCINE & SEROTYPES & VACCINE SEROTYPE & PERCENTAGE \\
\hline PCV7 & 4 9V 14 19F 23F 18C 6B & $19 \mathrm{~F}$ & 15.9 \\
PHiD-CV10 & 4 9V 14 19F 23F 18C 6B 15 7F & $19 \mathrm{~F}$ & 15.9 \\
PCV13 & 4 9V 14 19F 23F 18C 6B 15 7F 3 19A 6A & 19F 3 & 32.9 \\
PCV23 & 12 3 4 5 6B 7F 8 9N 9V 10A 11A 12F & 3 10A 11A 19F & 45.7 \\
\hline
\end{tabular}

Resistance to some common antibiotics used in treatment of pneumococcal infections was quite high in the nasopharyngeal isolates. Beta lactams are the mainstay of treatment of $S$. pneumoniae infections and drugs of choice especially penicillin and amoxicillin, for both invasive and non invasive infections. Penicillin and amoxicillin resistance among the pneumococci isolates was $49 \%$ and $6.6 \%$ as shown in Figure 3. This penicillin re- 
sistance was higher compared to data from other studies such as Australia where penicillin resistance of $S$. pneumoniae was $31 \%$ [11].

Resistance of the $S$. pneumoniae isolates to trimethoprim-sulphamethoxazole in this study was $91.1 \%$ (Figure 3). This was much higher than the resistance to trimethoprim-sulphamethoxazole $S$. pneumoniae isolates from a study in Milan, Italy which was $23.9 \%$ [12]. Trimethoprim-sulphamethoxazole is a common antibiotic used in HIV patients, to prevent infection with Pneumocystis carinii. Hence exposing the bacteria to subtherapeutic levels of the drug promotes development of resistance. However several studies have been conducted using Active Bacterial Core Surveillance to determine if long-term use increases risk of infection with trimethoprim-sulphamethoxazole resistant strains of bacteria. The study concluded that long term therapy with trimethoprim-sulphamethoxazole does not increase HIV patients' chance of becoming infected with a trimethoprim-sulphamethoxazole resistant strain of S. pneumoniae [13].

Resistance prevalence to erythromycin was $17.7 \%$ which was comparable to that of United States at $16 \%$. However, this was lower than that of New Zealand which was 22.9\% (Hellen Heffernan et al., 2009) and much lower than that of data collected in Milan, Italy where resistance by nasopharyngeal S. pneumoniae isolates to erythromycin was $52.6 \%$ [12]. Resistance prevalence of the $S$. pneumoniae isolates to cefotaxime and ceftriaxone, which are the preferred initial empiric therapy for suspected pneumococcal meningitis [14], were $2.4 \%$ and $6.7 \%$. Resistance prevalence to cefotaxime by S. pneumoniae isolates $(2.4 \%)$ was lower than that of the New Zealand study was which 5.9\% [15]. Because of the low prevalence, cefotaxime and ceftriaxone may still have significant antimicrobial effect on the $S$. pneumoniae isolates. Twenty four percent of the isolates were resistant to clindamycin which has excellent activity against penicillin-resistant organisms and has been successfully (with or without rifampin) used in children with AOM [12].

The nasopharyngeal S. pneumoniae isolates had lower resistance to levofloxacin which is the preferred fluroquinolone to Ofloxacin at 5.5\% and 13.3\% (Figure 3). This resistance to levofloxacin was much higher to resistance rates of S. pneumoniae isolates in the United States where the resistance was 1.0\% [16]. This levofloxacin resistance had increased from $0.3 \%$ in 1998 to $1.0 \%$ in 2002 [16].

WHO recommends co-trimoxazole for prophylaxis in children infected with HIV or HIV exposed, from six weeks old, to help prevent opportunistic infections [17]. Co-trimoxazole resistance prevalence in this study was highest in children below twelve months at 59\%. The average prevalence for all the age groups was 55.5\% which was higher than the $38 \%$ resistance prevalence of $S$. pneumoniae isolates to co-trimoxazole in New Zealand [15].

Vancomycin and chloramphenicol were the only antibiotics the $S$. pneumoniae isolates had not formed a resistance to. Considering that vancomycin is one of the last lines of defense in antimicrobial therapy for drug resistant $S$. pneumoniae, it is important to monitor for any new developments in resistance.

In this study, $23.6 \%$ of the recruited children had recently been on antibiotic therapy and $22.6 \%$ of these children were S.pneumoniae carriers. S. pneumoniae susceptible to these antibiotics may have been cleared in the nasopharynx of the non carriers, while the carriers may have acquired the pneumococci from other siblings as this was one of the risk factors.

Eighty eight percent of the pneumococci carriers who were exposed to antibiotics had penicillin resistant $S$. pneumoniae, while $58 \%$ and $12 \%$ showed resistance to erythromycin and amoxicillin. Twenty two percent of the carriers who were on antibiotic therapy had multi drug resistant nasopharyngeal $S$. pneumoniae isolates. Multi drug resistance was observed in $64.8 \%$ of the pneumococci isolates and majority of these serotypes were serotype 35B, $19 \mathrm{~F}$ and $13.47 .8 \%$ of serotype $35 \mathrm{~B}$ showed multi drug resistance where $81 \%$ were penicillin resistant this is shown in Table 6. Only one of the multi drug serotype 35B was resistant to Erythromycin. Serotype 19F had $73.3 \%$ penicillin resistant strains and $60 \%$ of these were multi drug resistant. Sixty three percent of the serotype 3 was multi drug resistant and only one of these serotypes was susceptible to penicillin.

Three of all the multidrug resistant serotypes (13, 10A and 7C) were resistant to Amoxicillin. Two additional serotypes to serotype 19F, serotype 10A and 13 were among PCV13 vaccine serotypes. As shown in Table 6 serotype 19F, which is a vaccine serotype, had the highest penicillin resistance of $73.3 \%$ among all the isolates. It also had a multidrug resistance of $20 \%$ which was lower than in the New Zealand study where $52 \%$ of $19 \mathrm{~F}$ isolates were multidrug resistant [15]. Serotypes 13 and $10 \mathrm{~A}$ had $15 \%$ and $10 \%$ multi drug resistant isolates.

\section{Conclusion}

Carriage prevalence of nasopharyngeal S. pneumonia was lower than that of similar prevalence studies and this 
may have been due to high frequency of antibiotic exposure by the HIV infected children. This may also have reflected on the major $S$. pneumoniae serotypes isolated hence the low frequency of Synflorix ${ }^{\mathrm{TM}}$ pneumococcal vaccine serotypes. The high resistance by the $S$. pneumoniae isolates to commonly used antibiotics is also major concern hence monitoring antibiotic resistance patterns and enforcing appropriate empirical drug choices is important.

\section{References}

[1] Janoff, E.N., Breiman, R.F. and Daley, C.L. (1992) Pneumococcal Disease during HIV Infection: Epidemiologic, Clinical, and Immunologic Prospectives. Annals of Internal Medicine, 117, 314-324. http://dx.doi.org/10.7326/0003-4819-117-4-314

[2] O’Brien, K.L. and Nohynek, H. (2003) The WHO Pneumococcal Vaccine Trials Carriage Working Group: Standard Method for Detecting Upper Respiratory Carriage of Streptococcus pneumoniae. The Pediatric Infectious Disease Journal, 22, 133-140. http://dx.doi.org/10.1097/01.inf.0000048676.93549.d1

[3] Kaldor, J., Asznowicz, R. and Dwyer, B. (1998) Serotyping of Streptococcus pneumoniae by Latex Agglutination. Pathology Journal, 20, 45-47.

[4] Abdullahi, O., Nyiro, J., Lewa, P., Slack, M. and Scott, J.A.G. (2008) The Descriptive Epidemiology of Streptococcus pneumoniae Nasopharyngeal Carriage in Children and Adults in Kilifi District, Kenya. The Pediatric Infectious Disease Journal, 27, 59-64. http://dx.doi.org/10.1097/INF.0b013e31814da70c

[5] Bogaert, D., de Groot, R. and Hermans, P.W. (2001) Streptococcus pneumoniae Colonization: The Key to Pneumococcal Disease. Lancet Infectious Disease Journal, 20, 289-295.

[6] Ciftci, E., Dogru, U., Aysev, D., Ince, E., Guriz, H. and Aysev, U.D. (2001) Investigation of Risk Factors for Penicillin-Resistant Streptococcus pneumoniae Carriage in Turkish Children. Pediatrics International, 43, 385-390. http://dx.doi.org/10.1046/j.1442-200X.2001.01422.x

[7] Lloyd-Evans, N., O’Dempsey, T.J., Baldeh, I., Secka, O., Demba, E., Todd, J.E., McArdle, T.F., Banya, W.S. and Greenwood, B.M. (1996) Nasopharyngeal Carriage of Pneumococci in Gambian Children and in Their Families. Pediatric Infections Disease Journal, 15, 866-871. http://dx.doi.org/10.1097/00006454-199610000-00007

[8] Mackenzie, G.A., Leach, A., Carpentis, J., Fisher, J. and Morris, P. (2010) Epidemiology of Nasopharyngeal Carriage of Respiratory Bacterial Pathogens in Children and Adults; Cross Sectional Surveys in a Population with High Rates for Pneumococcal Diseases. BMC Infectious Diseases, 10, 304. http://dx.doi.org/10.1186/1471-2334-10-304

[9] Lynch 3rd, J.P. and Zhanel, G.G. (2010) Streptococcus pneumoniae: Epidemiology and Risk Factors, Evolution of Antimicrobial Resistance and Impact of Vaccines. Current Opinion in Pulmonary Medicine, 16, 217-225. http://dx.doi.org/10.1097/mcp.0b013e3283385653

[10] GAVI's Pneumo ADIP: Pneumococcal Regional Serotype Distribution for Pneumococcal AMC TPP (2008) Baltimore, MD: Department of International Health, Johns Hopkins Bloomberg School of Public Health.

[11] Australian Group on Antimicrobial Resistance, 2005.

[12] Marchisio, P., Princip, N. and Sorella, S. (2002) Etiology of Acute Otitis Media in Human Immunodeficiency Virus-Infected Children. Pediatric Infections Disease Journal, 15, 58-61. http://dx.doi.org/10.1097/00006454-199601000-00013

[13] Fry, A.M., Udeagu, C.N., Soriano-Gabarro, M. and Fridkin, S. (2002) Epidemiology of Invasive S. pneumoniae Infections in Children.

[14] Daneman, N., McGeer, A., Green, K. and Low, D. (2006) Macrolide Resistance in Bacteremic Pneumococcal Disease: Implications for Patient Management. Clinical Infectious Diseases, 43, 432-438.

[15] Heffernan, H., Morgan, J. and Woodhouse, R. (2009) Invasive Pneumococcal Disease in New Zealand.

[16] Pletz, M.W., Maus, U., Krug, N., Welte, T. and Lode, H. (2008) Pneumococcal Vaccines: Mechanism of Action, Impact on Epidemiology and Adaption of the Species. International Journal of Antimicrobial Agents, 32, 199-206.

[17] World Health Organization: Guidelines on Co-Trimoxazole Prophylaxis for HIV-Related Infections among Children, Adolescents and Adults in Resource-Limited Settings. http://www.who.int/hiv/pub/guidelines/ctx/en/index.html 